\title{
Elements of a New Set Theory Based Quantum Mechanics with Applications in High Energy Quantum Physics and Cosmology
}

\author{
Mohamed S. EI Naschie \\ Distinguished Professor, Department of Physics, Faculty of Science, University of Alexandria, Alexandria, Egypt
}

Email address:

msnaschie10@gmail.com

To cite this article:

Mohamed S. El Naschie. Elements of a New Set Theory Based Quantum Mechanics with Applications in High Energy Quantum Physics and Cosmology. International Journal of High Energy Physics. Vol. 4, No. 6, 2017, pp. 65-74. doi: 10.11648/j.ijhep.20170406.11

Received: November 5, 2017; Accepted: November 16, 2017; Published: November 24, 2017

\begin{abstract}
We outline the quintessence of a fairly new quantum theory based on transfinite set theory representing a synthesis of the work of J. von Neumann's pointless continuous geometry, A. Connes' noncommutative quantum geometry, Menger-Urysohn dimensional theory, Sir R. Penrose's fractal tiling universe, 'tHooft's renormalon as well as D. Shectman quasi crystals and Einstein-Kaluza-Klein spacetime theories. We conclude that the Aether is a real non-materialistic material and that is essentially what we commonly call spacetime which can be accurately described as the empty five dimensional set in a Penrose quasi crystal universe. Seen in the light of the above, empty spacetime can be the source of infinite clean energy.
\end{abstract}

Keywords: Quantum Mechanics, Von Neumann Continuous Geometry, Noncommutative Geometry, Quasi Crystals, Einstein Spacetime, Kaluza-Klein Spacetime, Golden Mean Number System, Bijection Formula, Menger-Urysohn Dimensional Theory, The Empty Set, The Zero Set, Nothingness, E-Infinity, El Naschie's Cantorian Spacetime

\section{Introduction}

In the present work we draw in a nutshell the outline of a new quantum mechanics based mainly on transfinite set theory and noncommutative geometry [1-49]. It turns out that such an undertaking is much simpler and by far more intuitive than what one would have initially expected [3551]. In fact tying together Penrose fractal tiling universe [28, 29, 31, 32] and Connes noncommutative geometry [15-19] using the deductive dimensional theory of Menger-Urysohn [1-7] and modern nonlinear dynamics and fractals, we are almost at our destination $[26,36]$. Once we are that far one recognizes almost immediately that the pre-quantum particle is nothing more or less than the zero set [46-48] which is described accurately and fixed completely by not one but two dimensions, namely a topological Menger-Urysohn zero dimension and a corresponding Hausdorff dimension $\phi$ where $\phi=(\sqrt{5}-1) / 2[11,12,36]$. This $\phi$ results from plugging the zero topological dimension into a bijection formula relating the said two dimensions to each other in a unique way which corresponds to the dimensional function devised by A. Connes based on earlier work by von Neumann on continuous geometry to describe the so called seemingly pathological quotient space $\mathrm{X}$ of Penrose fractal tiling universe [1] [31, 32]. In Figure 1 we exhibit extracts from pages 90, 92 and 93 of A. Connes' classical book [15] illuminating the afore mentioned point in his characteristically accurate, mathematical and yet lucid language. Similarly one can easily obtain an accurate description for the pre-quantum wave which turned out to be fixed by the topological Menger-Urysohn dimension of minus one, i.e. it is an empty set $[1,12]$ and therefore has a corresponding Hausdorff dimension equal to $\phi^{2}$. That means it is the cobordism of the quantum zero set particle and may be interpreted geometrically as its surface as well as being its compliment because $\phi+\phi^{2}=1$. We can make the situation more plausible by realizing that the zeros of the zero set fill the voids of the empty set and together form a unit set [26, $46,49]$. That way many quantum paradoxes evaporate in thin air as it becomes obvious that any measurement will convert 
an empty set quantum wave by the mere act of measurement into a non-empty zero set pre-quantum particle which is an astoundingly simple resolution of the mysterious so called state vector reduction of conventional quantum mechanics [48-51]. Treading this set theoretical road we will be able to reason why there are three different kinds of energy densities in our cosmos, namely the ordinary measurable energy given by half of Hardy's probability of quantum entanglement [38][42-44] of two quantum particles, i.e. $\phi^{5} / 2$ percent of the total density, or stated different, almost $4.5 \%$ of the total energy density only while the rest of almost $95.5 \%$ of the total energy belongs to the so called dark energy sector which, as we will show later on, consists in turn of two parts, namely the dark matter energy and the pure dark energy density [43, 44]. It then turns out that this dark energy is really nothing else but the energy of the pre-quantum wave and is given exactly by $5 \phi^{2} / 2$, i.e. the 95.5 percent mentioned earlier on $[43,44,46]$. Furthermore we can easily reason that this quantum wave dark energy is actually more or less identical to the energy stored in spacetime ergo the Aether [39] which is in turn nothing else but a five dimensional empty set with the topological volume $5 \phi^{2}$ where $\phi^{2}$ is the Hausdorff dimension of the empty set and 5 may be regarded as the Kaluza-Klein topological dimension of spacetime [46] or the topological mass of the dark energy section [41, 43, 44]. Unlike conventional quantum mechanics [11] our present theory endows a pre-quantum particle with a path, albeit a fractal like path with a Hausdorff dimension equal $1+\phi$ rather than the classical topological path dimension of a zero point, namely unity $[8,12,46]$. Thus the zero set sweeps in our $D=5+\phi^{3}$ fractal version of KaluzaKlein spacetime, a path with a fractal dimension equal $1+\phi$ [46]. Similarly the world sheet in our space does not result in $1+1=2$ like in string theory but is given by $(1+\phi)+(1)=2+\phi$. Consequently spacetime is also not given by $(2)(2)=(2)+(2)=4$, i.e. the dimensions of Einstein spacetime $\mathrm{D}=4[43,46]$ but is given by $(2+\phi)+(2+\phi)=5+\phi^{3} \quad$ which is the earlier mentioned dimensionality of the fractal version of Kaluza-Klein spacetime which enveloped the fractal Einstein spacetime given by $4+\phi^{3}[43,46]$. This $4+\phi^{3}$ is the result of the union or intersection of the inverse of the Hausdorff dimension of the zero set particle, i.e. $1 / \phi=1+\phi$ and the empty set wave, i.e. $1 / \phi^{2}=2+\phi$ where we have a relation similar to $(2)(2)=(2)+(2)=4$, namely $(1 / \phi)\left(1 / \phi^{2}\right)=(1 / \phi)+\left(1 / \phi^{2}\right)=4+\phi^{3}$. This fractal Einstein space is clearly four dimensional on all scales, i.e. every point in it is also four dimensional so that our $5+\phi^{3}$ is nothing but $1+\left(4+\phi^{3}\right)=5+\phi^{3}$ as discussed and reasoned in various earlier publications [42-50]. We stress once more that the sources and elements of the present synthesis is an amalgamation of the thinking and results of D. Shechtman's quasi crystals [29, 31, 52], its geometrical topological description by Penrose fractal tiling universe
[31] and its algebraic analytical description by A. Connes' noncommutative geometry [15-19] [12-44] which is based on von Neumann's pointless continuous geometry [24, 32] and where the Menger-Urysohn dimensional theory also plays a pivotal role. We added to that the bijection formula [28-42] to replace and extend Connes' dimensional function and made extensive use of the Menger-Urysohn deductive theory of dimensions [9-14] [26, 36] which resulted in a giant step forward in our understanding of the essential differences between zero, empty and nothing and by realizing that way that the empty set must be negative topological dimension starting at minus one [38-42]. We did not stop there but extended the notion of empty set using the concept of the degree of emptiness of an empty set introduced some time ago by the late B. Mandelbrot [39] and that way made a profound conclusion preventing us from confusing zero with empty and empty with nothingness so that in this respect, we can claim to have the highest rigour possible not only in physics but also in pure mathematics [39]. Finally we must acknowledge that without L. Hardy's incredible result about the probability of quantum entanglement being equal $\phi^{5}$, something of a surprise which was confirmed using a very accurate experimental set up [53-59] we could not possibly have believed so firmly in the role of number theory in physics and so in particular the role of the quasi crystals golden mean [61-62] and the associated golden mean number system in organizing not only the macro but also the micro cosmos [53-59] to an extent bordering on the surreal. Although it is demonstratively true, it is still hard to believe because it is looking almost as if miracles and reality are merging together at least from the point of view of science rather than from the point of view of humble humans. As usual we apologize to the prospective reader for the moderately large number of references [1-67] which was found to be necessary to make a very long story moderately short and we hope this will prove to be helpful rather than inconvenient. In this connection we draw the attention of the reader to the content of Refs. 65 and 66 which depart markedly from the conventional mainstream literature in using Dirac's criteria of harmony and beauty extensively as well as to Ref. 67 which shows how wrong mainstream science can occasionally be and unsuspectingly so.

\section{Connes' Dimensional Function and the E-Infinity Bijection Formula}

The bijection formula of E-infinity [36-59] is central to our present theory which as we will reason here shortly, is not only more convenient to use than Connes' dimensional function of Penrose universe [31-38] but is also much more general and much less restricted or confined to a model [38]. In all events the important point behind both mathematical formulas is to find the Hausdorff dimension corresponding to a certain topological dimension. This is essentially nothing more than generalizing Mauldin-William's random one dimensional Cantor set to $\mathrm{n}$ topological dimensions. In the case of Connes' work, the dimensional function is given as is 
well known by [58, 59]

$$
D=a+b \phi
$$

where $a, b \in Z$ and $\phi=(\sqrt{5}-1) / 2$ which deviates from Ref. 15 in using $\phi=0.61833989$ instead of $1 / \phi=1.61833989$ but this can be trivially shown to be equivalently the same formalism. A detailed mathematical explanation in the original words of the creator of the theory, Prof. A. Connes, can be seen in Figure 1. Now to find the zero set Hausdorff dimension corresponding to our pre-quantum particle we just need to set $a=0$ and $b=1$ so that we obtain $D(0)=0+(1)(\phi)=\phi$. That way it seems $a=0$ implies the zero topological dimension of the zero set. Next to find $D=-1$ of the empty set it is clear that $a=1$ and $b=-1$ would do the trick but then it seems $\mathrm{b}$ and not a represents the topological dimension minus one of the empty set [36, 39]:

$$
\begin{aligned}
D & =1+(-1)(\phi)=1-\phi \\
& =\phi^{2}
\end{aligned}
$$

where $\phi^{2}$ could be understood equally as a frequency of the appearing of a form or the probability corresponding to the empty set or alternatively its Hausdorff dimensions. This situation might therefore be confusing although in all cases we obtained the right result, namely $D=\phi$ which is the Hausdorff dimension of the zero measure random Cantor set of Mauldin-Williams [36] and $D=\phi^{2}$ which is the Hausdorff dimension of the fat fractal positive measure complimentary set of the same Mauldin-Williams random Cantor set. On the other hand in the economic and neat notation of the bijection formula of E-infinity theory, things are much more clear and confusion is eliminated completely. The bijection formula states that $[36,39]$

$$
d_{c}^{(n)}=(1 / \phi)^{n-1}
$$

Here $d_{c}^{\left({ }^{(n)}\right.}$ is simply the Hausdorff dimension corresponding to a Cantor set with $\mathrm{n}$ Menger-Urysohn topological dimensions. Thus it is generic and basically not dependent on Penrose fractal tiling universe nor noncommutative geometry but as we said earlier on simply generalizing a Mauldin-Williams one dimensional random Cantor set construction to any arbitrary Cantor set topological dimension including negative dimensions as we will see shortly. Now to obtain the Hausdorff dimension corresponding to an empty set we simply set $n=-1$ for the Menger-Urysohn topological dimension of the void, i.e. the empty set in the bijection formula and find [48-59]

$$
\begin{aligned}
d_{c}^{(-1)} & =(1 / \phi)^{-1-1} \\
& =(1 / \phi)^{-2} \\
& =\phi^{2}
\end{aligned}
$$

while for the zero set we proceed similarly and just set $n=0$ to find that $[36,59]$

$$
\begin{aligned}
d_{c}{ }^{(0)} & =(1 / \phi)^{0-1} \\
& =(1 / \phi)^{-1} \\
& =\phi
\end{aligned}
$$

exactly as should be but this time without any ambiguity and almost automatically, the correct result pops up [33-59]. We also see that within this formalism the fractal Einstein spacetime comes out generically without fuss as [36-59]

$$
\begin{aligned}
d_{c}^{(4)} & =(1 / \phi)^{4-1} \\
& =(1 / \phi)^{3} \\
& =(1 / \phi)^{2}+(1 / \phi) \\
& =(1 / \phi)^{2} x(1 / \phi) \\
& =4+\phi^{3} \\
& =4+\frac{1}{4+\frac{1}{4+\ldots . .}} \\
& =4.236067977
\end{aligned}
$$

Next we will attempt to make the fact that the topological inductive dimension of an empty set must be minus one as plausible as possible but not more than that [36-59].

\section{The Negative Topological Dimension of an Empty Set [50-59]}

Let us start with a 3D cube which are the dimensions of the border, i.e. the surface or the "neighbourhood" of this cube. These are clearly 6 surfaces which are two dimensions so that the dimensions are simply $3-1=2$. Let us repeat the same deduction for the surface of the cube and find out that the neighbourhood are four lines, i.e. dimension 1 which means $2-1=1$. The third step is the inquiry about the dimension of the borders of the lines and this is clearly the two end points with dimension equal $1-1=0$ so that so far all agree with common sense. Now comes the ingenious questions which Karl Menger and independently Paul Urysohn must have asked themselves which is: what is the dimension of the neighbourhood of a point? The answer is found by extrapolating the same equation $n-1$ to become $0-1=-1$ and that is why the neighbourhood of a point which is clearly an empty set in the sense of set theory must have a negative and this would also make sense in physics as we will see so that we are of the firm opinion that at a very deep level pure mathematics and pure physical reality are one and the same thing. To obtain the corresponding Hausdorff dimension we just need to set $n=-1$ in the bijection formula as shown 
earlier on. Now we notice that the empty set has a positive Hausdorff dimension $\phi^{2}$ and is thus far from being nothing, so we can ask ourselves why stop here? Why not go on and ask what is the neighbourhood dimension of the empty set? The answer is easy because at least formally we can go on with our inductive formula and see that the topological MengerUrysohn dimension must be $-1-1=-2$ and setting this in the bijection formula, i.e. $n=-2$ one finds an even emptier set than the classical empty set, namely [36-59]

$$
\begin{aligned}
d_{c}^{(-2)} & =(1 / \phi)^{-2-1} \\
& =(1 / \phi)^{3} \\
& =\phi^{3}
\end{aligned}
$$

This is a highly interesting result related to Mandelbrot's notion of the degree of emptiness of the empty set because inverting $\phi^{3}$ one finds the fractal Einstein spacetime dimension $4+\phi^{3}=1 / \phi^{3}$. That clearly suggests that the surface of the pre-quantum wave $\phi^{2}$ is spacetime itself and that spacetime is really a multi-fractal starting by the surface of the empty set which we can now equate with the Aether and then go on with increasing negative dimensions, i.e. -1 , $-2, \ldots .$. to minus infinity. However here we find at long last a meaning for the word Nothingness. That we do by setting $n=-\infty$ and find that at this point the corresponding Hausdorff dimension truly vanishes because [54-59] $-\infty-1=-\infty$ gives

$$
\begin{aligned}
d_{c}^{(-\infty)} & =(1 / \phi)^{-\infty-1} \\
& =(1 / \phi)^{-\infty} \\
& =\phi^{\infty} \\
& =\text { zero }
\end{aligned}
$$

This is the real insubstantial nothingness and by contrast spacetime, the Aether, the zero set and the empty is far from being nothing. They are something. This should settle the great discussion about nothingness that is central to the lecture and subsequent discussion of F. Wilczek and L. Krauss [51].

\section{Implications for Ordinary Energy and Dark Energy [56, 58, 59]}

To have something rather than nothing we surely need volume. This self evident fact can be taken here as an axiom above proof, also for a Cantorian spacetime made entirely of union and intersection of infinitely many elementary MauldinWilliams Cantor sets which our E-infinity spacetime is made of. In our theory we use the core of this space with $D=5+\phi^{3}$ [48, 59]. Seeing things from such a perspective it is understandable that we sought to devise a non-classical notion for volume which is appropriate for a zero measure Cantor set. Since the topological dimension as well as the measure of a Mauldin-Williams random Cantor set is zero, we have nothing there to indicate that it is there except its Hausdorff dimension $\phi$ [48-59]. Consequently we extend the notion of volume to a Hausdorff-topological volume found by intersection, i.e. a multiplicative volume. In five topological dimensions this is simply $\phi^{5}$. This move is known in the mathematical theory of measure as the volume interpretation of the Hausdorff dimension. Remarkably this happened to be the Hardy probability of quantum entanglement of two quantum particles. Remembering that the zero set $(0, \phi)$ model the quantum particle we could now move to calculate the corresponding kinetic energy density in this case to be [57-59]

$$
\begin{aligned}
E(O) & =[\text { volume of }(0)]\left[1 / 2 m(v \rightarrow c)^{2}\right] \\
& =\left(\phi^{5}\right)(1 / 2)\left(m c^{2}\right) \\
& =\left(\phi^{5} / 2\right)(\text { Einstein maximal energy density })
\end{aligned}
$$

In other words the density of the ordinary measurable energy of the quantum particle is [57-59]

$$
\begin{aligned}
\gamma(O) & =\phi^{5} / 2 \\
& \cong 1 / 22 \\
& \cong 4.5 \%
\end{aligned}
$$

of the total maximal density $\gamma(\max )=1$ of Einstein's famous formula $E=\mathrm{mc}^{2}$ interpreted in the spirit of our previous elucidation of this subject. Needless to repeat again that this is in astonishing agreement with all modern accurate measurements and cosmic observation as reported in countless publications [57-59]. From the above it is a trivial matter to conclude that dark energy density, i.e. the energy which is supposedly missing in the cosmos must be equal to one minus what we just found as ordinary measurable energy density of the zero set quantum particle [55-59], i.e.

$$
\begin{aligned}
\gamma(D) & =1-\gamma(O) \\
& =1-\phi^{2} / 2
\end{aligned}
$$

This turns out to be exactly equal [55-59]

$$
\gamma(D)=5 \phi^{2} / 2
$$

It is not difficult to interpret this value as the energy density of the quantum wave empty set in analogy to the energy density of the zero set quantum particle as follows: The $5 \phi^{2} / 2$ is clearly an additive Hausdorff-topological volume corresponding to the multiplicative $\phi^{5}$, i.e. $5 \phi^{2} / 2$ is the surface of $\phi^{5}$ or its cobordism while the factor $(1 / 2)$ is coming from the same Newtonian kinetic energy we saw while deriving $\gamma(O)=\phi^{5} / 2$. The entire derivation is 
naturally consistent with the fact that $\phi^{2}$ belongs to the empty set quantum wave in the same way as $\phi$ is connected to the zero set quantum particle as amply demonstrated earlier on using the bijection formula $[36,59]$. In the course of time we have given in the past five years or so different mathematical derivations of both the ordinary energy and the dark energy density of the cosmos with exactly the same final result stated above. However we could go further and show how the dark section which cannot be measured directly at present because of phenomena of wave collapse and the limitation imposed at present by the non-existence of nondemolition measurement devises consists in fact of two different parts, namely an attractive dark matter section containing $22 \%$ of the total energy density and a repulsive pure dark energy density of about $73 \%$ of the total $[43,44]$.

In conclusion of this section we may mention another subtle derivation of the various cosmic energy that proceeds direct from Newtonian kinetic and potential energy. This derivation rests on the understanding that ordinary energy has a Hausdorfftopological mass equal $\phi^{3}$ while the corresponding dark energy mass is given by the topological Kaluza-Klein mass $m=5$ [59]. In addition we have the topological velocity of light being $c=\phi$ as will be discussed in the next section of the present work. Proceeding on this basis we find [59]

$$
\begin{aligned}
\gamma(O) & =1 / 2 m(v \rightarrow c)^{2} \\
& =1 / 2 \phi^{3}(c=\phi)^{2} \\
& =\phi^{5} / 2
\end{aligned}
$$

and

$$
\begin{aligned}
\gamma(O) & =1 / 2 m(v \rightarrow c)^{2} \\
& =1 / 2(5)\left(\phi^{2}\right) \\
& =5 \phi^{2} / 2
\end{aligned}
$$

exactly as we expected. Using Newton potential energy the same result is obtain as shown on previous occasions [59]. Incidentally obtaining the same result using kinetic energy of the motion or the potential energy of rest indicates that at this deep level of mechanics, zenon paradox about the illusive nature of motion is philosophically not misguided [60].

\section{Maxwell Equation of Electromagnetism and the Topological Speed of Light [53]}

One of the most remarkable features of the Maxwell equations [53] is that the Einstein constancy of the speed of light may be deduced from two dual fundamental constants of these arguably most fundamental and beautiful equations of physics. Denoting the permittivity constant of free space by $\varepsilon_{o}$ and the corresponding permeability constant with $\mu_{o}$, the speed of light is found to be [53]

$$
c=\frac{1}{\sqrt{\mu_{o} \varepsilon_{o}}}
$$

It is not difficult to see that using the above we can now refute, based on the very same fundamental arguments, that at quantum scales the speed of light is constant but variable with an expectation value. To explain that, we first have to remember a few vital points. The first point is that calculus is at the root of Maxwell's equations while we know very well from the work of G. Ord, L. Nottale [4-7] as well as the Cantorian-E-infinity proposals [54] that calculus gives a false picture in general of the fine structure details of a theory and it is therefore not recommended in looking at non-classical mechanics. We say that in full knowledge and understanding that the Schrödinger equations as well as the Dirac equations are obtained using calculus. However by the same token we know that the exact results of Hardy regarding $\phi^{5}$ was obtained using Dirac's ket and bra method and not any other formalism involving calculus and taking the limit [54]. Second the total symmetry between electric charges and magnetic charges are broken because classically we have no monopoles in Maxwell's original classical equations. However monopoles are conjectured and invoked in high energy physics and unification theories and 'tHooft's polycove magnetic monopole is just one example. Consequently at a very high energy we expect that the constancy of the speed of light would be at a minimum debatable. Taking all these points on board and remembering that we have a relative permittivity which is $\varepsilon_{r}=1$ for the vacuum, then we could go on and postulate that in fractal Cantorian spacetime $\varepsilon_{r}$ would not represent the resistance to a classical line path of a quantum particle but the fractal path of the same which means we can set $\varepsilon_{r}=1+\phi$ and by a complete symmetry after admitting monopoles, we can set the analogous relative constant $\mu_{r}=1+\phi$. Inserting in the speed of light formula of Maxwell, one finds the topological speed of light to be given by [53]

$$
\begin{aligned}
c(\text { topological }) & =\frac{1}{\sqrt{\varepsilon_{r} \mu_{r}}} \\
& =\frac{1}{\sqrt{(1+\phi)(1+\phi)}} \\
& =\phi
\end{aligned}
$$

exactly as deduced using different arguments in previous publications $[59,60]$. 
$X$ as an ordinary space, one sees very quickly that the classical tools do not work, and do not distinguish $X$ from the space consisting of a single point. For example, given two tilings $T_{1}$ and $T_{2}$ and any finite portion $P$ of $T_{1}$, one can find exactly the same configuration $P$ occurring in $T_{2}$; thus no finite portion enables one to distinguish $T_{1}$ from $T_{2}$ ([Gru-S]). Thus any configuration which does occur in some tiling $T$, such as those of Figure 3, will occur (and infinitely many times) in any other tiling $T^{\prime}$. This geometric property translates into the triviality of the topology of $X$. The natural topology on $X$ has for closed sets $F \subset X$ the closed sets of $K$ that are saturated for $\mathcal{R}$. But, every equivalence class for $\mathcal{R}$ is dense in $K$, and it follows that the only closed sets $F \subset X$ are $F=\varnothing$ and $F=X$. Thus the topology of $X$ is trivial and does not distinguish $X$ from a point; it is, of course, not Hausdorff and it contains no interesting information.

One possible attitude toward such an example would be to say that, up to fluctuations, there is only one tiling $T$ of the plane and not to be disturbed by the distinction between $X$ and a point. However, we shall see that $X$ is a very interesting "noncommutative" space or "quantum" space, and that one of its topological invariants, the dimension group, is the subgroup of $\mathbb{R}$ generated by $\mathbb{Z}$ and the golden number $\frac{1+\sqrt{5}}{2}$. This will show, in particular, why the density, or frequency of appearance, of a motif in the tiling must, entirely on account of the topology of the set of all tilings, be an element of the group $\mathbb{Z}+\left(\frac{1+\sqrt{5}}{2}\right) \mathbb{Z}$.

the semigroup $\mathbb{N}$ of nonnegative integers to the ordered group $\left(\mathbb{Z}, \mathbb{Z}^{+}\right)$of integers.

This ordered group is very easy to calculate for finite-dimensional algebras such as the algebras $A_{n}$, and for the algebra $A=\overline{U A_{n}}$ encountered earlier. Since $A_{n}$ is the direct sum of two matrix algebras, we have

$$
K_{0}\left(A_{n}\right)=\mathbb{Z}^{2}, K_{0}\left(A_{n}\right)^{+}=\mathbb{Z}^{+} \oplus \mathbb{Z}^{+} \subset \mathbb{Z} \oplus \mathbb{Z} .
$$

The ordered group $\left(K_{0}(A), K_{0}(A)^{+}\right)$is then the inductive limit of the ordered groups $\left(\mathbb{Z} \oplus \mathbb{Z}, \mathbb{Z}^{+} \oplus \mathbb{Z}^{+}\right)$, the inclusion of the $n$th into the $(n+1)$ st being given by the matrix

$$
\left[\begin{array}{ll}
1 & 1 \\
1 & 0
\end{array}\right]
$$

which corresponds to the inclusion $A_{n} \subset A_{n+1}$ described earlier.

Since this matrix defines a bijection $(a, b) \rightarrow(a+b, a)$ of $\mathbb{Z}^{2}$ onto $\mathbb{Z}^{2}$ the desired inductive limit is the group $K_{0}(A)=\mathbb{Z}^{2}$. However, this bijection is not a bijection of $\mathbb{Z}^{+} \oplus \mathbb{Z}^{+}$onto $\mathbb{Z}^{+} \oplus \mathbb{Z}^{+}$, and in the limit the semigroup $K_{0}(A)^{+}$becomes $K_{0}(A)^{+}=\left\{(a, b) \in \mathbb{Z}^{2} ;\left(\frac{1+\sqrt{5}}{2}\right) a+b \geq 0\right\}$, as one sees on diagonalizing the matrix $\left[\begin{array}{ll}0 & 1 \\ 1 & 1\end{array}\right]$ (Figure 4 ).

It follows that, module the choice of basis of $\underline{\underline{Z}}^{2}$, i.e. modulo $\operatorname{PSL}(2, \mathbb{Z})$, the golden number appears as a topological invariant of the space $X$ through the $C^{*}$-algebra $A$. In another formulation, one shows that this $C^{*}$-algebra has a unique trace $\tau$. Thus there exists a unique linear form $\tau$ on $A$ such that $\tau(x y)=\tau(y x)$ for all $x, y \in A_{1}$ and $\tau(1)=1$. The values of $\tau$ on the projections form the intersection $\left(\mathbb{Z}+\frac{1+\sqrt{5}}{2} \mathbb{Z}\right) \cap \mathbb{R}_{+}$. This trace $\tau$ is positive, i.e., satisfies

$$
\tau\left(a^{*} a\right) \geq 0 \quad(\forall a \in A)
$$

and it may be calculated for $a=a_{\left(z, z^{\prime}\right)}$ directly as the integral of the diagonal entries of the matrix $a$ :

$$
\tau(a)=\int_{K} a_{(z, z)} \mathrm{d} \mu(z),
$$

where the probability measure $\mu$ on $K$ is uniquely determined by the condition $\tau(a b)=\tau(b a)(\forall a, b \in A)$. In classical measure theory, given a Radon measure $\rho$ on a compact space $Y$, the Hilbert space $L^{2}(Y, \rho)$ is obtained as the completion of $C(Y)$ for the scalar product $\langle f, g\rangle=\int f \bar{g} \mathrm{~d} \rho$, and $L^{\infty}(Y, \rho)$ is the weak closure of the algebra $C(Y)$ acting by multiplication on $L^{2}(Y, \rho)$. In the case that we are interested in, the algebra $A$ replaces $C(Y)$, the positive

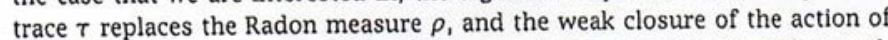
$A$ by left multiplication on $L^{2}(A, \tau)$ (the completion of $A$ for the scalar product $\left.\langle a, b\rangle=\tau\left(a^{*} b\right)\right)$ is the hyperfinite factor of type $\mathrm{II}_{1}$ of Murray and von Neumann $[$ Mur-N], which we shall denote by $R$. 


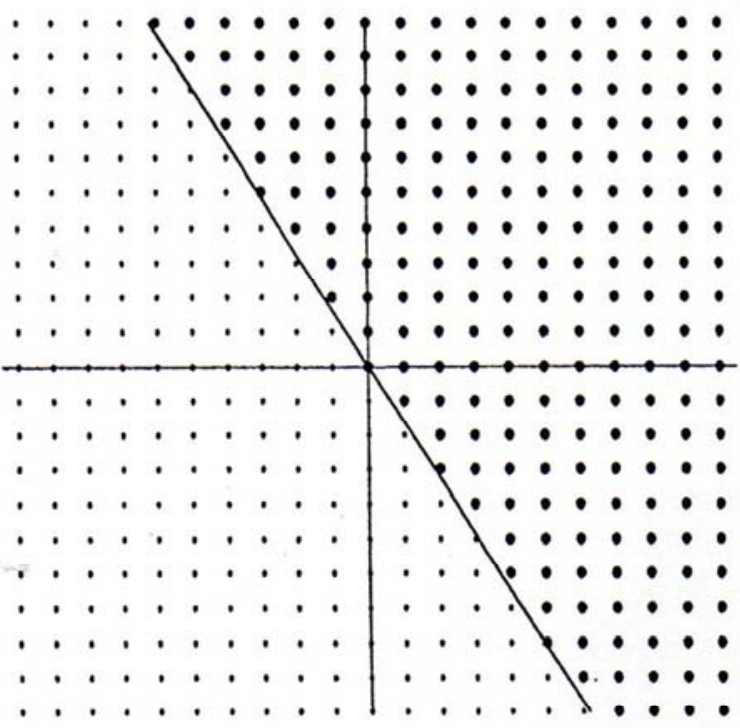

Figure II.4. $K_{0}^{+}(A)=\left\{(n, m) \in \mathbb{Z}^{2} ; n\left(\frac{1+\sqrt{5}}{2}\right)+m \geq 0\right\}$

Whereas the continuous dimension of Murray and von Neumann can take a all the positive real values for the projections of $R$ (or of the matrix rings $\left.I_{n}(R)\right)$ since $\operatorname{dim}(e)=T(e)$ for the projections that belong to $A$ this dimension an only take values in the subgroup $\mathbb{Z}+\left(\frac{1+\sqrt{5}}{2}\right) \mathbb{Z}$, which accounts for the role of bese numbers in measuring the densities of tiles, or of patterns of a given type a a generic Penrose tiling, in accordance with our interpretation in Chapter I if the continuous dimensions as densities. To summarize, we have shown in his example that the "topology" of $X$ is far from being trivial, that it gives rise o the $C^{*}$-algebra $A$, which is a simple $C^{*}$-algebra, uniquely characterized as a **algebra (up to Morita equivalence) by the properties

1) $A$ is the inductive limit of finite-dimensional algebras; it is said to be approximately finite (or $A F$ ).

2) $\left(K_{0}(A), K_{0}(A)^{+}\right)=\left(\mathbb{Z}^{2},\left\{(a, b) ;\left(\frac{1+\sqrt{5}}{2}\right) a+b \geq 0\right\}\right)$.

Finally, we note that the $C^{*}$-algebra $A$ is exactly the one that appears in the :onstruction by Vaughan Jones [Jone ${ }_{2}$ ] of subfactors of index less than 4 , for ndex equal to the golden ratio (Chapter V, Section 10). This can be exploited o describe explicitly the factor in this geometric situation.

Figure 1. Three excerpts from pages 90, 92 and 93 of A. Connes' classical book [15] explaining the dimensional function of noncommutative geometry of a Penrose universe.

\section{Conclusion}

The present work was not meant to be complete or even a comprehensive account of our mildly ambitious attempt to produce a new quantum physics based on set theory using numerous elements of the work of Maxwell, Einstein Tesla, von Neumann, Connes, Penrose, Shectman, Menger, Kaluza, Klein, Urysohn, 'tHooft, Gross, Witten, Mauldin and the vast literature on the golden mean number system as a transfinite
Turing machine with infinite capacity to do calculation [110]. Going this road we have at a minimum a real feeling that we are now near to the old dream of being able to build a reactor for free energy from empty space. This is no more just wishful thinking particularly after recognizing the reality of the Aether as a non-materialist material with real existence. Much of the courage to speak out about our conclusions regarding this point stems in no minor measure from a superb lecture and subsequent discussion by a truly beautiful mind of theoretical physics, Frank Wilczek who 
was presented and interviewed by an exceptional science communicator Laurence Krauss [51]. Anyone who saw the video of this event must at the end ask himself how come we sometimes overlook the obvious to that extent? This is a very interesting question for the philosopher and historian of science and something which we intend to come back to in future if the "force" remains with us and permits it but at present a new, simple and elegant quantum mechanics not borne out of the gloom of the Weimar republic [63] but rather from the optimism which sees the universe as a self similar quasi crystal [61, 62] golden topology and a geometry which, as surmised long ago by Paul Dirac [64], can explain the hostility of the great Einstein towards a non-geometrical quantum theory by presenting for the first time a geometrical non-commutative theory for the quantum based on a golden super computer code of our universe [50].

\section{References}

[1] Ji-Huan He and M. S. El Naschie: A collection of publications on E-infinity Cantorian spacetime theory. Transfinite Physics. China Culture \& Science, Shanghai, 2005. Publishing, 2005. ISBN 988-98846-5-8.

[2] P. Weibel, G. Ord, O. Rössler (Editors): Spacetime Physics and Fractality. Festschrift in honour of Mohamed El Naschie on the occasion of his $60^{\text {th }}$ birthday. Springer, Vienna-New York (2005).

[3] Ji-Huan He, E. Goldfain, L. D. Sigalotti and A. Mejias and M. S. El naschie: Beyond the 2006 Physics Nobel Prize for COBE. An introduction to E-infinity spacetime theory. China Sci. \& Culture Publishing, Shanghai. (2006). ISBN: 98807681-9.4/0.4.

[4] G. Ord (Guest Editor): Transfinite Physics-Treading the path of Cantor and Einstein. Chaos, Solitons \& Fractals, 25(4), 2005.

[5] Mohamed S. El Naschie, L. Nottale, S. Al Athel and G. Ord: Fractal space-time and Cantorian geometry in quantum mechanics. Chaos, Solitons \& Fractals, 7(6), 1996.

[6] M. S. El Naschie, O. E. Rössler and G. Ord: Chaos, information and diffusion in quantum physics. Chaos, Solitons \& Fractals, 7(5), 1996.

[7] M. S. El Naschie, O. E. Rössler and I. Prigogine Editors): Quantum Mechanics, Diffusion and Chaotic Fractals. Pergamon Press/Elsevier, Oxford, 1995.

[8] M. S. El Naschie: Quantum two-slit gedanken experiment revisited. In "Einstein in Alexandria". Editor, W. Witten. Bibliotheca Alexandrina Publishing, Alexandria, Egypt, 2008, pp. 141-153. ISBN 977-6163-49-1.

[9] M. S. El Naschie: Gödel, Cantor and Modern Nonlinear Dynamics: In 'First International Symposium on Gödel's Theorem's', Editor Z. W. Wolkowski. World Scientific, Singapore, 1993, pp. 95-106.

[10] M. S. El Naschie: On a general theory for quantum gravity. In 'Science of the Interface. Editor H. Diebner, T. Druckrey and P. Weibel. Genista Verlag, Tübingen, Germany, 2001, pp. 875880 .
[11] M. S. El Naschie: Deterministic quantum mechanics versus classical mechanical indeterminism and nonlinear dynamics. In "Frontiers of Fundamental Physics" Editors: B. Sidharth, A. Alfonso-Faus and Marius J. Fullana I Alfonso. American Institute of Physics, AIP Conference Proceedings No. 905, New York, 2007, pp. 56-66.

[12] M. S. El Naschie: Complexity theory interpretation of high energy physics and elementary particle mass spectrum. In "Frontiers of Fundamental Physics" Editors: B. Sidharth. University Press, Hyderabad, India, Vol. 3, New York, 2007, pp. 1-32. ISBN: 9788173715778.

[13] M. S. El Naschie: 'tHooft dimensional regularization implies transfinite heterotic string theory and dimensional transmutation. In "Frontiers of Fundamental Physics" Editors: B. Sidharth and M. V. Altaisky, Kluwer Academic/Plenium Publisher, New York, USA, Vol. 4, 2001, pp. 81-86.

[14] M. S. El Naschie: The Cantorian gravity coupling constant is $\alpha_{\mathrm{gs}}(\max )=1 / 26.18033989$. In "Frontiers of Fundamental Physics" Editors: B. Sidharth and M. V. Altaisky, Kluwer Academic/Plenium Publisher, New York, USA, Vol. 4, 2001, pp. 87-95.

[15] A. Connes: Noncommutative Geometry. Academic Press, San Diego, USA, 1994.

[16] F. Scheck, H. Upmeier and W. Werner (Editors): Noncommutative Geometry and The Standard Model of Elementary Particle Physics. Springer, Berlin, Germany, 1996.

[17] G. Landi: An Introduction to Noncommutative Spaces and Their Geometrics. Springer, Berlin, 1997.

[18] A. Connes and M. Marcolli: Noncommutative Geometry, Quantum Fields and Motives. American Mathematical Society, Rhode Island, USA, 2008.

[19] Shahn Majid (Editor): On space and time. Cambridge Press, Cambridge, UK, 2008.

[20] J-Pierre Changeux and Alain Connes: Conversations on Mind, Matter and Mathematics. (Editor and translated by M. B. Debevoise). Princeton University Press, Princeton, New Jersey, USA, 1989.

[21] HRH The Prince of Wales, Tony Juniper and Ian Skelly: Harmony: A New Way of Looking At Our World. Harper Collins Publications, London, UK. 2010. (see in particular page 113).

[22] A. Connes, A. Lichnerowicz and M. P. Schützenberger: Triangle of Thought. American Mathematical Society, Providence, Rhode Island, USA, 2001.

[23] M. Waldschmidt, P. Moussa, J. M. Luck and C. Itzykson (Editors): From Number Theory to Physics. Springer Verlag, Berlin, Germany, 1992.

[24] J. von Neumann: Continuous Geometry, Vol. 25. Princeton University Press, Princeton, New Jersey, USA, 1988.

[25] V. Kodiyalam and V. S. Sunder: Topological Quantum Field Theories From Subfactors. Chapman \& Hall/CRC, London, UK, 2001.

[26] M. S. El Naschie: Superstrings, knots and noncommutative geometry in E-infinity space. International Journal of Theoretical Physics, 37(12), 1998, pp. 2935-2951. 
[27] J. Madore: An Introduction to Noncommutative Differential Geometry and Its Physical Applications. Cambridge University Press, Cambridge, UK, 1999.

[28] L. Marek-Crnjac: Generalized quantum entanglement family in connection to black holes and nanotechnology. Chapter in "Quantum Gravity", Edited by B. Mitchell. Nova Publishers, New York, USA. 2017, pp. 33-48.

[29] L. Marek-Crnjac: Cantorian Space-Time Theory: The Physics of Empty Sets in Connection With Quantum Entanglement and Dark Energy. Lambert Academic Publishing, Saarbrucken, Germany, 2013. ISBN: 978-3-65912876-9.

[30] Y. Manin: Topics in Noncommutative Geometry. Princeton University Press, Princeton, USA. 2014.

[31] M. S. El Naschie: Penrose universe and Cantorian spacetime as a model for noncommutative quantum geometry. Chaos, Solitons \& Fractals, 9(6), 1998, p. 931-933.

[32] M. S. El Naschie: von Neumann geometry and E-infinity quantum spacetime. Chaos, Solitons \& Fractals, 9(12), 1998, pp. 2023-2030.

[33] M. S. El Naschie: Nuclear spacetime theories, superstrings, monster group and applications, Chaos, Solitons \& Fractals, 10(2-3), 1999, pp. 567-580.

[34] M. S. El Naschie: Einstein's dream and fractal gravity. Chaos, Solitons \& Fractals, 24(1), 2005, pp. 1-5.

[35] Mohamed S. El Naschie: The Quantum Gravity Immirzi Parameter-A General Physical and Topological Interpretation. Gravity and Cosmology, 19(3), 2013, 151-153.

[36] M. S. El Naschie: A review of E-infinity theory and the mass spectrum of high energy particle physics. Chaos, Solitons \& Fractals, 19(1), 2004, pp. 209-236.

[37] M. S. El Naschie: On the uncertainty of Cantorian geometry and the two-slit experiment. Chaos, Solitons \& Fractals, 19(3), 1998, pp. 517-529.

[38] Mohamed S El Naschie: The self similarity equivalence relation connecting Newton's energy with Einstein's energy and dark energy. International Journal of Innovation in Science and Mathematics, 4(1), 2016, pp. 42-57.

[39] Mohamed S. El Naschie: The Aether of spacetime physics is the empty set of pure mathematics. Natural Science, 9(9), 2017, pp. 289-292.

[40] Mohamed S. El Naschie: Spacetime as a new frontier advanced material with applications in physics, engineering, chemistry and cosmology. Advanced Material Physics and Chemistry, 7(9), 2017, pp. 347-352.

[41] Mohamed S El Naschie: From a dual Einstein-Kaluza spacetime to "tHooft renormalon and the reality of accelerated cosmic expansion. Journal of Modern Physics, 8(8), 2017, pp. 1319-1329.

[42] Mohamed S. El Naschie: Spacetime from Zitterbewegung. Open Journal of Modelling and Simulation, 5(3), 2017, pp. 169-173.

[43] Mohamed S. El Naschie: Einstein-Kaluza combined spacetime as the optimal and simplest framework to compute and understand dark matter, pure dark energy and measurable ordinary energy. Natural Science, 9(8), 2017, pp. 241-244.
Letter to the Editor.

[44] Mohamed S. El Naschie: A combined Heterotic string and Kähler manifold elucidation of ordinary energy, dark matter, Olbers's paradox and pure dark energy density of the cosmos. Journal of Modern Physics, 8(7), 2017, pp. 11011118 .

[45] Mohamed S. El Naschie: The looped light of the triple-slit real experiment as a confirmation for the extra dimensions of quantum spacetime and the reality of dark energy. Optical and Photonic Journal, 7(2), 2017, pp. 19-26.

[46] Mohamed S. El Naschie: On a fractal version of Witten's Mtheory. Journal of Astronomy \& Astrophysics, 6(2), 2016, pp. 135-144.

[47] A. J. Babchin and M. S. El Naschie: On the real Einstein beauty $\mathrm{E}=\mathrm{kmc}^{2}$. World Journal of Condensed Matter Physics, 6(1), 2016, pp. 1-6.

[48] Mohamed S. El Naschie: The emergence of spacetime from the quantum in three steps. Advances in Pure Mathematics, 6(6), 2016, pp. 446-454.

[49] Mohamed S. El Naschie: Looped light on dark energy. Journal of Quantum Information Science, 7(2), 2017, pp. 43-47.

[50] Mohamed S. El Naschie and Scott Olsen: The universe as a golden super computer. YouTube:

https://www.youtube.com/watch?v=3GiyWK_JQxg. 1 October 2017.

[51] F. Wliczek and L. Krauss: Materiality of a vacuum (YouTube https://www.youtube.com/Watch?V=BBXDrNn6PVg). 24 February 2017.

[52] Dan Monroe: Nobel Prize-Discovery of Quasi Crystals Physics-October 7, 2011. Physics Review Focus 28, 14. https://physics.aps.org/story/V28/st14.

[53] R. Penrose: The Road to Reality. J. Cape, London, UK. 2004.

[54] Mohamed S. El Naschie: Quantum entanglement as a consequence of a Cantorian micro spacetime geometry. Journal of Quantum Information Science, 1(2), 2011, pp. 5053.

[55] Mohamed S. El Naschie: Three quantum particles Hardy entanglement from the topology of Cantorian-fractal spacetime and the Casimir effect as dark energy-A great opportunity for nanotechnology. American Journal of Nano Research and Applications, 2015, 3(1), pp. 1-5.

[56] Mohamed S. El Naschie: A resolution of cosmic dark energy via quantum entanglement relativity theory. Journal of Quantum Information Science, 2013, 3, pp. 23-26.

[57] M. S. El Naschie, From $\mathrm{E}=\mathrm{mc}^{2}$ to $\mathrm{E}=\mathrm{mc}^{2} / 22-\mathrm{A}$ short account of the most famous equation in physics and its hidden quantum entangled origin. Journal of Quantum Information Science, 4, 2014, pp. 284-291.

[58] L. Marek-Crnjac, Mohamed S. El Naschie:, Ji-Huan He: Chaotic Fractals at the Root of Relativistic Quantum Physics and Cosmology. International Journal of Modern Nonlinear Theory and Application, 2(1A), 2013, pp. 78-88.

[59] Mohamed S. El Naschie: On a non-perturbative quantum relativity theory leading to a Casimir-dark energy nanotech reactor proposal. Open Journal of Applied Science, 5(7), 2015, pp. 313-324. 
[60] Mohamed S. El Naschie: On a non-perturbative quantum relativity theory leading to a Casimir-dark energy nanotech reactor proposal. Open Journal of Applied Science, 5(7), 2015, pp. 313-324.

[61] Mohamed S. El Naschie: The Gap Labelling Integrated Density of States For a Quasi Crystal Universe Is Identical to The Observed 4.5 Percent Ordinary Energy Density of The Cosmos. Natural Science, 6, 2014, 1259-1265.

[62] G. Iovane: El Naschie E-infinity Cantorian spacetime and length scales in cosmology. International Journal of Nonlinear Sciences and Numerical Simulation, 7(2), 2006, pp. 155-162.

[63] P. Forman: Weimar Culture, Causality and Quantum Theory. Historical Studies in Physical Sciences, University of California Press, California, USA, 3, 1971, pp. 1-115.
[64] P. A. M. Dirac: Quotation taken from the Introduction of Prof. J. Madore's book of Ref. 52 based on citation in The Mathematical Intelligence, 11, 1989, pp. 58.

[65] M. S. El Naschie: An outline for a quantum golden field theory. Chaos, Solitons \& Fractals, 37(2), 2008, pp. 317-323.

[66] M. S. El Naschie: Quantum golden field theory-Ten theorems and various conjectures. Chaos, Solitons \& Fractals, 36, 2008, pp. 1121-1125.

[67] M. S. El Naschie: Forbidden symmetries, Cantor sets and hypothetical graphite. Chaos, Solitons \& Fractals, 4(12), 1994, pp. 2269-2272. 